\title{
Spiral laser beams in inhomogeneous media
}

\author{
Alex Mahalov, ${ }^{1}$ Erwin Suazo, ${ }^{1,2}$ and Sergei K. Suslov ${ }^{1, *}$ \\ ${ }^{1}$ School of Mathematical and Statistical Sciences, Arizona State University, Tempe, Arizona 85287-1804, USA \\ ${ }^{2}$ School of Mathematical Sciences, University of Puerto Rico, Mayaguez, Puerto Rico 00681-9000, USA \\ ${ }^{*}$ Corresponding author: sks@asu.edu
}

Received June 3, 2013; accepted June 16, 2013;

posted June 18, 2013 (Doc. ID 191472); published July 25, 2013

\begin{abstract}
Explicit solutions of the inhomogeneous paraxial wave equation in a linear and quadratic approximation are applied to wave fields with invariant features, such as oscillating laser beams in a parabolic waveguide and spiral light beams in varying media. A similar effect of superfocusing of particle beams in a thin monocrystal film, harmonic oscillations of cold trapped atoms, and motion in magnetic field are also mentioned. (c) 2013 Optical Society of America

OCIS codes: (000.3860) Mathematical methods in physics; (080.0080) Geometric optics; (080.2710) Inhomogeneous optical media.

http://dx.doi.org/10.1364/OL.38.002763
\end{abstract}

Green function and generalized Fresnel integrals. In the context of quantum mechanics, a one-dimensional (1D) linear Schrödinger equation for generalized driven harmonic oscillators,

$$
\begin{aligned}
i \psi_{t}= & -a(t) \psi_{x x}+b(t) x^{2} \psi-i c(t) x \psi_{x} \\
& -i d(t) \psi-f(t) x \psi+i g(t) \psi_{x},
\end{aligned}
$$

$(a, b, c, d, f$, and $g$ are suitable real-valued functions of time $t$ only), can be solved by the integral superposition principle:

$$
\psi(x, t)=\int_{-\infty}^{\infty} G(x, y, t) \psi(y, 0) \mathrm{d} y
$$

where

$$
\begin{aligned}
G(x, y, t)= & {\left[2 \pi \mu_{0}(t)\right]^{-1 / 2} } \\
& \times \exp \left[i \left(\alpha_{0}(t) x^{2}+\beta_{0}(t) x y\right.\right. \\
& \left.\left.+\gamma_{0}(t) y^{2}+\delta_{0}(t) x+\varepsilon_{0}(t) y+\kappa_{0}(t)\right)\right],
\end{aligned}
$$

for certain initial data $\psi(x, 0)=\varphi(x)$ (see [1-ㅁ] and the references therein for more details).

The intrinsic connection between Hamiltonian mechanics and the process of wave propagation is anything but a new idea [5,6]. Yet, in paraxial optics, when the time variable $t$ represents the coordinate, say $s$, in the direction of wave propagation, Eqs. (2) and (3) can be thought of as a generalization of the Fresnel integral [7-10].

In the paraxial approximation, a 2D coherent light field in a parabolic inhomogeneous medium with coordinates $(\mathbf{r}, s)=(x, y, s)$ is described by the following equation for the complex field amplitude:

$$
\begin{aligned}
i A_{s}= & -a\left(A_{x x}+A_{y y}\right)+b\left(x^{2}+y^{2}\right) A \\
& -i c\left(x A_{x}+y A_{y}\right)-2 i d A \\
& -\left(x f_{1}+y f_{2}\right) A+i\left(g_{1} A_{x}+g_{2} A_{y}\right),
\end{aligned}
$$

where $a, b, c, d, f_{1,2}$, and $g_{1,2}$ are real-valued functions of the coordinate in the direction of wave propagation $s$. The latter equation can be reduced to the standard form

$$
-i \chi_{\tau}+\chi_{\xi \xi}+\chi_{\eta \eta}=c_{0}\left(\xi^{2}+\eta^{2}\right) \chi,
$$

$\left(c_{0}=0,1\right)$ by the following Ansatz:

$$
A=\mu^{-1} e^{i\left(\alpha\left(x^{2}+y^{2}\right)+\delta_{1} x+\delta_{2} y+\kappa_{1}+\kappa_{2}\right)} \chi(\xi, \eta, \tau),
$$

where $\xi=\beta(s) x+\varepsilon_{1}(s), \eta=\beta(s) y+\varepsilon_{2}(s)$, and $\tau=\gamma(s)$ (see Lemma 1 of [10] for a detailed statement).

The corresponding 2D Fresnel integral for inhomogeneous media in the linear and quadratic approximation is obtained in [10] (which may include intensity fluctuations from a random phase modulation). The GaussianHermitian beams are given by separation of the variables

$$
\begin{aligned}
A_{n m}(\mathbf{r}, s)= & \frac{e^{i\left(\kappa_{1}+\kappa_{2}\right)+2 i(n+m+1) \gamma}}{\sqrt{2^{n+m} n ! m ! \pi}} \beta \\
& \times e^{i\left(\alpha\left(x^{2}+y^{2}\right)+\delta_{1} x+\delta_{2} y\right)-\left(\beta x+\varepsilon_{1}\right)^{2} / 2-\left(\beta y+\varepsilon_{2}\right)^{2} / 2} \\
& \times H_{n}\left(\beta x+\varepsilon_{1}\right) H_{m}\left(\beta y+\varepsilon_{2}\right),
\end{aligned}
$$

in terms of solutions of certain Ermakov-type systems, which are known in quadratures [2] [see Eqs. (9)-(14) below for an important explicit special case].

Oscillating and breathing laser beams. For a 1D paraxial wave equation with quadratic refractive index,

$$
2 i A_{s}+A_{x x}-x^{2} A=0,
$$

an important class of Gaussian-Hermitian modes can be presented as follows:

$$
\begin{aligned}
A_{n}(x, s)= & e^{i\left(\alpha x^{2}+\delta x+\kappa\right)+i(2 n+1) \gamma} \sqrt{\frac{\beta}{2^{n} n ! \sqrt{\pi}}} \\
& \times e^{-(\beta x+\varepsilon)^{2} / 2} H_{n}(\beta x+\varepsilon),
\end{aligned}
$$

where $H_{n}(x)$ are the Hermite polynomials [11] and

$$
\begin{gathered}
\alpha(s)=\frac{\alpha_{0} \cos 2 s+\sin 2 s\left(\beta_{0}^{4}+4 \alpha_{0}^{2}-1\right) / 4}{\beta_{0}^{4} \sin ^{2} s+\left(2 \alpha_{0} \sin s+\cos s\right)^{2}}, \\
\beta(s)=\frac{\beta_{0}}{\sqrt{\beta_{0}^{4} \sin ^{2} s+\left(2 \alpha_{0} \sin s+\cos s\right)^{2}}},
\end{gathered}
$$




$$
\begin{gathered}
\gamma(s)=-\frac{1}{2} \arctan \frac{\beta_{0}^{2} \tan s}{1+2 \alpha_{0} \tan s}, \\
\delta(s)=\frac{\delta_{0}\left(2 \alpha_{0} \sin s+\cos s\right)+\varepsilon_{0} \beta_{0}^{3} \sin s}{\beta_{0}^{4} \sin ^{2} s+\left(2 \alpha_{0} \sin s+\cos s\right)^{2}} \\
\varepsilon(s)=\frac{\varepsilon_{0}\left(2 \alpha_{0} \sin s+\cos s\right)-\beta_{0} \delta_{0} \sin s}{\sqrt{\beta_{0}^{4} \sin ^{2} s+\left(2 \alpha_{0} \sin s+\cos s\right)^{2}}} \\
\kappa(s)=\sin ^{2} s \frac{\varepsilon_{0} \beta_{0}^{2}\left(\alpha_{0} \varepsilon_{0}-\beta_{0} \delta_{0}\right)-\alpha_{0} \delta_{0}^{2}}{\beta_{0}^{4} \sin ^{2} s+\left(2 \alpha_{0} \sin s+\cos s\right)^{2}} \\
+\frac{1}{4} \sin 2 s \frac{\varepsilon_{0}^{2} \beta_{0}^{2}-\delta_{0}^{2}}{\beta_{0}^{4} \sin ^{2} s+\left(2 \alpha_{0} \sin s+\cos s\right)^{2}}
\end{gathered}
$$

The real- or complex-valued parameters $\alpha_{0}, \beta_{0} \neq 0$, $\gamma_{0}=0, \delta_{0}, \varepsilon_{0}$, and $\kappa_{0}=0$ are initial data of the corresponding Ermakov-type system $[2, \underline{2}, \underline{12}, 13]$. A direct Mathematica verification can be found in Media 1. (Harmonic motion of cold trapped atoms is experimentally realized [14].)

These explicit solutions that are omitted in all textbooks on quantum mechanics (see $[\underline{13}, \underline{15}]$ ) provide a new multiparameter family of oscillating Gaussian-Hermitian beams in parabolic (self-focusing fiber) waveguides, which deserve an experimental observation; special cases were theoretically studied earlier in [8,16]. Examples are shown on Figs. $\underline{1}$ and $\underline{2}$. (Particular solutions in terms of Airy functions can be obtained in analogy with $[\underline{5}, \underline{17}-19]$.)

Spreading solutions. The homogeneous paraxial wave equation,

$$
2 i B_{s}+B_{x x}=0
$$

can be transformed by the substitution

$B(x, s)=\frac{1}{\left(s^{2}+1\right)^{1 / 4}} \exp \left(\frac{i s x^{2}}{2\left(s^{2}+1\right)}\right) A\left(\frac{x}{\sqrt{s^{2}+1}}, \arctan s\right)$,

into the inhomogeneous form in Eq. (7) (see [12] and the references therein). Composition of Eqs. (8) and (16) results in multiparameter solutions to parabolic Eq. ( $\underline{15})$ :

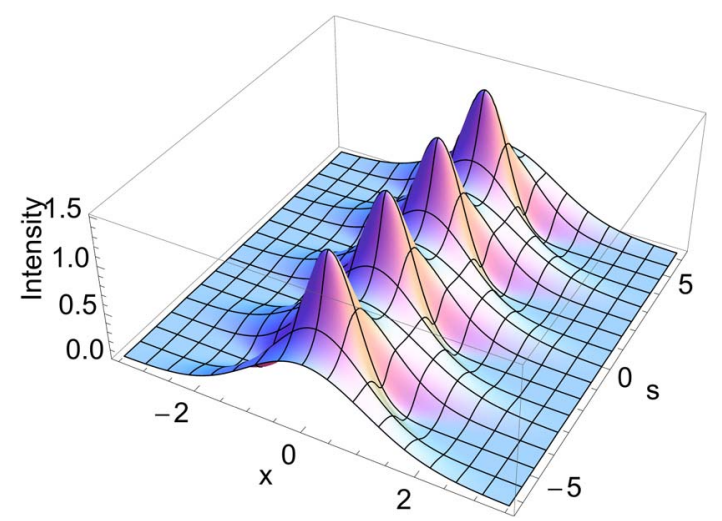

Fig. 1. Breathing Gaussian mode.

$$
\begin{aligned}
B_{n}(x, s)= & {\left[\left(\left(2 \alpha_{0} s+1\right)^{2}+\beta_{0}^{4} s^{2}\right)\right]^{-1 / 4} } \\
& \times \sqrt{\frac{\beta_{0}}{2^{n} n ! \sqrt{\pi}}} \exp \left(\frac{i x^{2}\left(\left(4 \alpha_{0}^{2}+\beta_{0}^{4}\right) s+2 \alpha_{0}\right)}{2\left(\left(2 \alpha_{0} s+1\right)^{2}+\beta_{0}^{4} s^{2}\right)}\right) \\
& \times \exp \left(i x \frac{\left(2 \alpha_{0} s+1\right) \delta_{0}+s \beta_{0}^{3} \varepsilon_{0}}{\left(2 \alpha_{0} s+1\right)^{2}+\beta_{0}^{4} s^{2}}\right) \\
& \times \exp \left(i s \frac{\left(2 \alpha_{0} s+1\right)\left(\beta_{0}^{2} \varepsilon_{0}^{2}-\delta_{0}^{2}\right)-2 s \beta_{0}^{3} \delta_{0} \varepsilon_{0}}{2\left(\left(2 \alpha_{0} s+1\right)^{2}+\beta_{0}^{4} s^{2}\right)}\right) \\
& \times \exp \left(-i\left(n+\frac{1}{2}\right) \arctan \left(\frac{\beta_{0}^{2} s}{2 \alpha_{0} s+1}\right)\right) \\
& \times \exp \left(-\frac{\left(\beta_{0}\left(x-\delta_{0} s\right)+\varepsilon_{0}\left(2 \alpha_{0} s+1\right)\right)^{2}}{2\left(\left(2 \alpha_{0} s+1\right)^{2}+\beta_{0}^{4} s^{2}\right)}\right) \\
& \times H_{n}\left(\frac{\beta_{0}\left(x-\delta_{0} s\right)+\varepsilon_{0}\left(2 \alpha_{0} s+1\right)}{\sqrt{\left(2 \alpha_{0} s+1\right)^{2}+\beta_{0}^{4} s^{2}}}\right) .
\end{aligned}
$$

Their direct Mathematica verification is also provided in Media 1 (see also []]).

Breathing spiral laser beams. By the Ansatz $\Psi(X, Y, T)=\chi(\xi, \eta, \tau), T=-\tau$, and

$$
\left(\begin{array}{l}
X \\
Y
\end{array}\right)=\left(\begin{array}{cc}
\cos \omega \tau & -\sin \omega \tau \\
\sin \omega \tau & \cos \omega \tau
\end{array}\right)\left(\begin{array}{l}
\xi \\
\eta
\end{array}\right)
$$

( $\omega=$ constant), Eq. (5) with $c_{0}=1$ can be transformed to the equation of motion for the isotropic planar harmonic oscillator in a perpendicular uniform magnetic field (in the rotating frame of reference):

$$
i \Psi_{T}+\Psi_{X X}+\Psi_{Y Y}=\left(X^{2}+Y^{2}\right) \Psi+i \omega\left(X \Psi_{Y}-Y \Psi_{X}\right) .
$$

The latter equation was solved in the early days of quantum mechanics by Fock $[\underline{20}, \underline{21}]$ in polar coordinates, $X=R \cos \Theta$ and $Y=R \sin \Theta$ :

$$
\begin{aligned}
\Psi(R, \Theta, T)= & \sqrt{\frac{n !}{\pi(n+|m|) !}} e^{-i E T} \\
& \times e^{i m \Theta} R^{|m|} e^{-R^{2} / 2} L_{n}^{|m|}\left(R^{2}\right), \\
E & =4 n+2(|m|+1)-m \omega,
\end{aligned}
$$

( $m= \pm 0, \pm 1, \ldots, n=0,1, \ldots)$ in terms of Laguerre polynomials [11]. This wave function coincides, up to

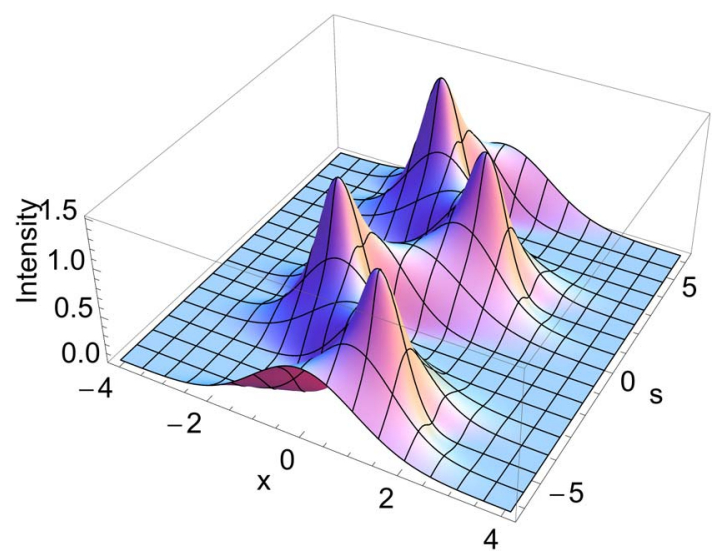

Fig. 2. Bending and breathing Gaussian mode. 
a simple factor, with the one for a flat isotropic oscillator without magnetic field. Therefore, its development in terms of Eq. (6) for standard harmonics is a 2D special case of the multidimensional expansions from [11] (see also $[22,23]$ and the references therein).

As a result, by back substitution one arrives at a general family of spiral solutions in inhomogeneous media. For example, the 2D paraxial wave equation $(\omega=0)$,

$$
2 i A_{s}+A_{x x}+A_{y y}=\left(x^{2}+y^{2}\right) A,
$$

possesses the following Gaussian-Laguerre modes:

$$
\begin{aligned}
A_{n}^{m}(x, y, s)= & \beta \sqrt{\frac{n !}{\pi(n+m) !}} \\
& \times e^{i\left(\alpha\left(x^{2}+y^{2}\right)+\delta_{1} x+\delta_{2} y+\kappa_{1}+\kappa_{2}\right)} e^{i(2 n+m+1) \gamma} \\
& \times\left(\beta(x \pm i y)+\varepsilon_{1} \pm i \varepsilon_{2}\right)^{m} e^{-\left(\beta x+\varepsilon_{1}\right)^{2} / 2-\left(\beta y+\varepsilon_{2}\right)^{2} / 2} \\
& \times L_{n}^{m}\left(\left(\beta x+\varepsilon_{1}\right)^{2}+\left(\beta y+\varepsilon_{2}\right)^{2}\right), \quad m \geq 0,
\end{aligned}
$$

by the explicit action of Schrödinger's group (see $[10,12]$ and the references therein for classical accounts). Here, Eqs. (9) through (14) are utilized for real or complex parameters $\alpha_{0}, \beta_{0} \neq 0, \delta_{0}^{(1,2)}$, and $\varepsilon_{0}^{(1,2)}$ (the last two sets may be different). Examples are shown in Figs. $\underline{3}$ and $\underline{4}$.

Spreading and rotating solutions. The homogeneous parabolic equation,

$$
2 i B_{s}+B_{x x}+B_{y y}=0,
$$

and Eq. (21) are related by the transformation

$$
\begin{aligned}
B(x, y, s)= & \frac{1}{\left(s^{2}+1\right)^{1 / 2}} \exp \left(\frac{i s\left(x^{2}+y^{2}\right)}{2\left(s^{2}+1\right)}\right) \\
& \times A\left(\frac{x}{\sqrt{s^{2}+1}}, \frac{y}{\sqrt{s^{2}+1}}, \arctan s\right) .
\end{aligned}
$$

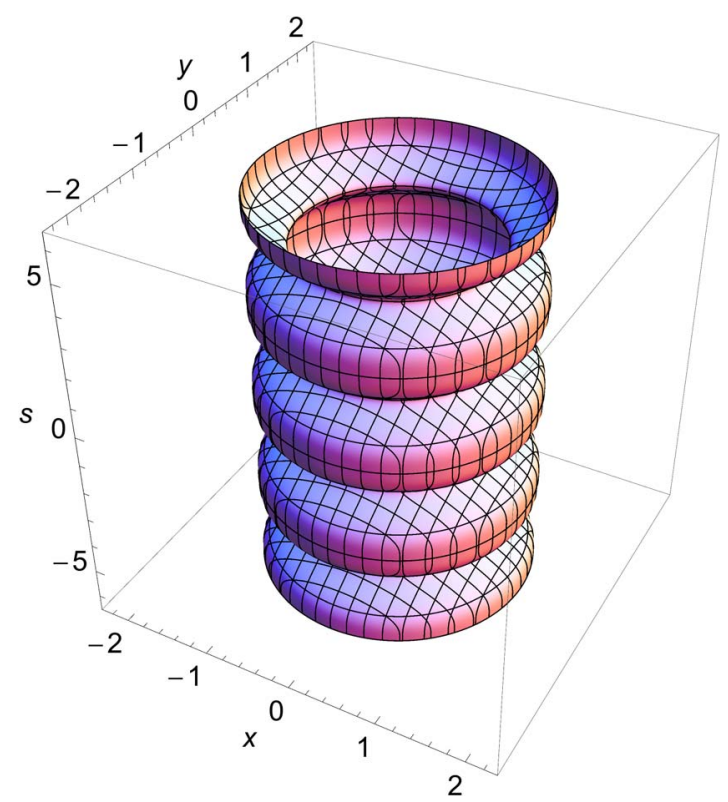

Fig. 3. Breathing Gaussian mode: surface where the intensity $|A|^{2}$ changes by the factor $e$.

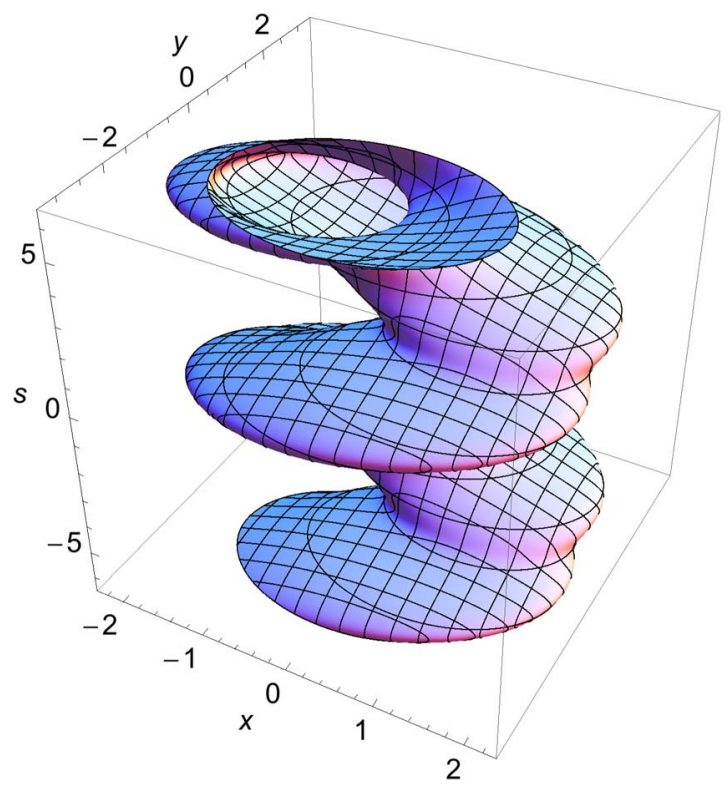

Fig. 4. Breathing and rotating Gaussian mode: surface where the intensity $|A|^{2}$ changes by the factor $e$.

Examples of spiral laser beams in a uniform medium are discussed in [24-26] (see also $[\underline{8}, 16]$ ).

A multiparameter solution is given by

$$
\begin{aligned}
B_{n}^{m}(x, y, s)= & \frac{e^{i s\left(\delta_{0}^{(1)^{2}}+\delta_{0}^{(2)^{2}}\right) /(2(1+2 \alpha s))}}{\sqrt{\left(2 \alpha_{0} s+1\right)^{2}+\beta_{0}^{4} s^{2}}} \\
& \times \exp \left(-i(1+m+2 n) \arctan \left(\frac{s \beta_{0}^{2}}{1+2 \alpha_{0} s}\right)\right) \\
& \times \exp \left(i \frac{\alpha_{0}\left(x^{2}+y^{2}\right)+x \delta_{0}^{(1)}+y \delta_{0}^{(2)}}{2 \alpha_{0} s+1}\right) \\
& \times \exp \left[-\frac{\left(\beta_{0}\left(x-\delta_{0}^{(1)} s\right)+\varepsilon_{0}^{(1)}\left(2 \alpha_{0} s+1\right)\right)^{2}}{2\left(2 \alpha_{0} s+1+i \beta_{0}^{2} s\right)\left(1+2 \alpha_{0} s\right)}\right] \\
& \times \exp \left[-\frac{\left(\beta_{0}\left(y-\delta_{0}^{(2)} s\right)+\varepsilon_{0}^{(2)}\left(2 \alpha_{0} s+1\right)\right)^{2}}{2\left(2 \alpha_{0} s+1+i \beta_{0}^{2} s\right)\left(1+2 \alpha_{0} s\right)}\right] \\
& \times\left[\frac{\beta_{0}(x+i y)-\left(\delta_{0}^{(1)}+i \delta_{0}^{(2)}\right) s}{\sqrt{\left(2 \alpha_{0} s+1\right)^{2}+\beta_{0}^{4} s^{2}}}\right. \\
& \left.+\frac{\left(\varepsilon_{0}^{(1)}+i \varepsilon_{0}^{(2)}\right)\left(2 \alpha_{0} s+1\right)}{\sqrt{\left(2 \alpha_{0} s+1\right)^{2}+\beta_{0}^{4} s^{2}}}\right]^{m} \\
& \times L_{n}^{m}\left[\frac{\left(\beta_{0}\left(x-\delta_{0}^{(1)} s\right)+\varepsilon_{0}^{(1)}\left(2 \alpha_{0} s+1\right)\right)^{2}}{\left(2 \alpha_{0} s+1\right)^{2}+\beta_{0}^{4} s^{2}}\right. \\
& \left.+\frac{\left(\beta_{0}\left(y-\delta_{0}^{(2)} s\right)+\varepsilon_{0}^{(2)}\left(2 \alpha_{0} s+1\right)\right)^{2}}{\left(2 \alpha_{0} s+1\right)^{2}+\beta_{0}^{4} s^{2}}\right] .
\end{aligned}
$$

A similar effect of superfocusing of proton beams in a thin monocrystal film was discussed in [27] (validity of the 2D harmonic crystal model had been confirmed by Monte Carlo computer experiments). Among other quantum mechanical analogs, the minimum-uncertainty squeezed states for atoms and photons in a cavity are 
reviewed in [28]. (See also [6,10,19,29] and the references therein for extensions to nonlinear geometrical optics; an optoacoustic experiment is proposed in [30].)

In summary, we present multiparameter solutions to homogeneous and inhomogeneous paraxial wave equations which may be of interest in adaptive optics of (partially) coherent beams propagating through an atmospheric turbulence $[\underline{17}, \underline{31}-\underline{33}]$ and deserve an experimental observation.

We thank Eugeny Abramochkin and Christoph Koutschan for help and Kamal Barley for graphics enhancement. This research was partially supported by AFOSR grant FA9550-11-1-0220.

\section{References}

1. R. Cordero-Soto, R. M. Lopez, E. Suazo, and S. K. Suslov, Lett. Math. Phys. 84, 159 (2008).

2. N. Lanfear, R. M. López, and S. K. Suslov, J. Russ. Laser Res. 32, 352 (2011).

3. E. Suazo and S. K. Suslov, J. Russ. Laser Res. 33, 63 (2012).

4. S. K. Suslov, Proc. Am. Math. Soc. 140, 3067 (2012).

5. V. A. Fock, Electromagnetic Diffraction and Propagation Problems (Pergamon, 1965).

6. S. N. Vlasov and V. I. Talanov, Radiophys. Quantum Electron. 38, 1 (1995).

7. M. Born and E. Wolf, Principles of Optics, 7th ed. (Pergamon, 1999).

8. M. B. Vinogradova, O. V. Rudenko, and A. P. Sukhorukov, Theory of Waves (Nauka, 1979) [in Russian].

9. V. V. Dodonov and V. I. Man'ko, in Invariants and the Evolution of Nonstationary Quantum Systems (Nova Science, 1989), p. 103.

10. A. Mahalov and S. K. Suslov, "Solution of paraxial wave equation for inhomogeneous media in linear and quadratic approximation," Proc. Am. Math. Soc. (to be published).

11. A. F. Nikiforov, S. K. Suslov, and V. B. Uvarov, Classical Orthogonal Polynomials of a Discrete Variable (Springer, 1991).
12. R. M. López, S. K. Suslov, and J. M. Vega-Guzmán, Phys. Scr. 87, 038112 (2013).

13. R. M. López, S. K. Suslov, and J. M. Vega-Guzmán, J. Differ. Equ. Appl. 19, 543 (2013).

14. D. Leibfried, R. Blatt, C. Monroe, and D. Wineland, Rev. Mod. Phys. 75, 281 (2003).

15. M. E. Marhic, Lett. Nuovo Cimento Soc. Ital. Fis. 22, 376 (1978).

16. G. P. Agrawal, A. K. Ghatak, and C. L. Mehtav, Opt. Commun. 12, 333 (1974).

17. Y. Gu and G. Gbur, Opt. Lett. 35, 3456 (2010).

18. E. G. Abramochkin and E. Razueva, Opt. Lett. 36, 3732 (2011).

19. A. Mahalov and S. K. Suslov, Phys. Lett. A 377, 33 (2012).

20. V. Fock, Zs. für Phys. 47, 446 (1928).

21. V. A. Fock, Selected Works: Quantum Mechanics and Quantum Field Theory (Chapman \& Hall/CRC, 2004), p. 29.

22. M. Meiler, R. Cordero-Soto, and S. K. Suslov, J. Math. Phys. 49, 072102 (2008).

23. R. Cordero-Soto and S. K. Suslov, Theor. Math. Phys. 162, 286 (2010).

24. R. Piestun, Y. Y. Schechner, and J. Shamir, J. Opt. Soc. Am. 17, 294 (2000).

25. E. G. Abramochkin and V. G. Volostnikov, Phys. Usp. 47, 1177 (2004).

26. M. R. Hatzvi and Y. Y. Schechner, Opt. Lett. 37, 3207 (2012).

27. Y. N. Demkov, Phys. Atomic Nuclei 72, 779 (2009).

28. S. I. Kryuchkov, S. K. Suslov, and J. M. Vega-Guzmán, J. Phys. B 46, 104007 (2013).

29. E. A. Kuznetsov and S. K. Turitsyn, Phys. Lett. A 112, 273 (1985).

30. A. Y. Okulov, J. Phys. B 41, 101001 (2008).

31. O. Korotkova, N. Farwell, and A. Mahalov, Waves Random Media 19, 692 (2009).

32. X. Pang, G. Gbur, and T. D. Visser, Opt. Lett. 36, 2492 (2011).

33. Y. Gu, J. Opt. Soc. Am. A 30, 708 (2013). 